\title{
Homogenization of a quasi-linear problem with quadratic growth in perforated domains: An example
}

by

\author{
Juan CASADO-DÍAZ \\ Departamento de Ecuaciones Diferenciales y Análisis Numérico, \\ Facultad de Matemáticas, C. Tarfía s/n, 41012 Sevilla, Spain. \\ E-mail: jcasado@numer.us.es
}

ABSTRACT. - We study the homogenization of Dirichlet problems for a fixed quasi-linear operator which is the perturbation of the Laplace operator by the square of the gradient, when the domain varies arbitrarily. With respect to the Dirichlet problem for the linear Laplace operator posed on the same domains, a new nonlinear zeroth order term appears in the homogenized problem. We also give a corrector result.

Key words: Quasi-linear problem, perforated donain.

RÉsumÉ. - On étudie l'homogénéisation de problèmes de Dirichlet pour un opérateur quasi-linéaire fixe qui est la perturbation de l'opérateur de Laplace par le carré du gradient, pour une suite de domaines qui varient arbitrairement. Par rapport au problème de Laplace avec des conditions de Dirichlet posé sur les mêmes domaines, il apparaît un nouveau terme non-linéaire d'ordre zéro dans le problème homogénéisé. On obtient aussi un résultat de correcteur.

\section{INTRODUCTION}

We consider in the present paper the following homogenization problem: Let $\Omega_{n}$ be a sequence of open sets which are included in a fixed bounded open set $\Omega$ of $\mathbb{R}^{d}$. For $\gamma, \lambda \in \mathbb{R},(\lambda>0)$ and for $f \in L^{\infty}(\Omega)$, we consider

A.M.S. Classification: 35 B 27, 35 B 40.

Annales de l'Institut Henri Poincaré - Analyse non linéaire - 0294-1449 
the solution $u_{n}$ of the problem:

$$
\left\{\begin{array}{l}
-\Delta u_{n}+\lambda u_{n}=f+\gamma\left|\nabla u_{n}\right|^{2} \text { in } \mathcal{D}^{\prime}\left(\Omega_{n}\right) \\
u_{n} \in H_{0}^{1}\left(\Omega_{n}\right) \cap L^{\infty}\left(\Omega_{n}\right) .
\end{array}\right.
$$

The existence of a solution for this type of problems has been proved in [4] and its uniqueness in [1] (In the present case, it can also easily he obtained by the change of unknown fontion (0.5) below). It is also shown in [4] that the norm of $u_{n}$ in $H_{0}^{1}\left(\Omega_{n}\right) \cap L^{\infty}\left(\Omega_{n}\right)$ is a bounded sequence in $\mathbb{R}$. Identifying the function $u_{n}$ with its extension by zero in $\Omega \backslash \Omega_{n}$, we conclude that $u_{n}$ is a bounded sequence in $H_{0}^{1}(\Omega) \cap L^{\infty}(\Omega)$ and so, extracting a subsequence, we deduce that $u_{n}$ converges to a function $u$, weakly in $H_{0}^{1}(\Omega)$ and weakly-* in $L^{\infty}(\Omega)$. Our problem is to find the equation satisfied by the function $u$.

The answer to this homogenization problem is well known in the linear case (see [9], [11], [12], [10]) where the problem satisfied by $u_{n}$ is now

$$
\left\{\begin{array}{l}
-\Delta u_{n}=f \text { in } \mathcal{D}^{\prime}\left(\Omega_{n}\right) \\
u_{n} \in H_{0}^{1}\left(\Omega_{n}\right)
\end{array}\right.
$$

In this linear case, $u_{n}$ is still bounded in $H_{0}^{1}(\Omega)$ and there exists a nonnegative measure $\mu$ which vanishes on the sets of zero capacity such that $u_{n}$ converges in $H_{0}^{1}(\Omega)$ weakly to the unique solution $u$ of the homogenized equation

$$
\left\{\begin{array}{l}
u \in H_{0}^{\mathrm{j}}(\Omega) \cap L_{\mu}^{2}(\Omega) \\
\int_{\Omega} \nabla u \nabla v d x+\int_{\Omega} u v d \mu=\int_{\Omega} f v d x \\
\forall v \in H_{0}^{1}(\Omega) \cap L_{\mu}^{2}(\Omega)
\end{array}\right.
$$

In the case where $\mu$ is a Radon measure, the functions of $\mathcal{D}(\Omega)$ belong to $L_{\mu}^{2}(\Omega)$. This implies that the solution of $(0.3)$ satisfies

$$
\left\{\begin{array}{l}
-\Delta u+\mu u=f \text { in } \mathcal{D}^{\prime}(\Omega) \\
u \in H_{0}^{1}(\Omega) \cap L_{\mu}^{2}(\Omega) .
\end{array}\right.
$$

Therefore, the problem satisfied by the function $u$ is not yet $(0.2)$, and a new term, $\mu u$, appears. Let us emphasize that this term only depends on the values of $u$, and not of its gradient. It is moreover linear with respect to $u$.

To carry out the homogenization of $(0.1)$, the idea is to make the change of unknown function

$$
z_{n}=e^{\gamma u_{n}}-1
$$


The new problem we obtain is nothing but

$$
\left\{\begin{array}{l}
-\Delta z_{n}=\left(f-\lambda u_{n}\right) \gamma e^{\gamma u_{n}} \text { in } \mathcal{D}^{\prime}\left(\Omega_{n}\right) \\
z_{n} \in H_{0}^{1}\left(\Omega_{n}\right) \cap L^{\infty}\left(\Omega_{n}\right),
\end{array}\right.
$$

in which we can pass to the limit using the result of the linear case. Coming back to the old unknown functions, we will prove that the function $u$ now satisfies the homogenized equation

$$
\left\{\begin{array}{l}
u \in H_{0}^{1}(\Omega) \cap L^{\infty}(\Omega) \cap L_{\mu}^{2}(\Omega) \\
\int_{\Omega} \nabla u \nabla v d x+\lambda \int_{\Omega} u v d x+\frac{1}{\gamma} \int_{\Omega} \frac{e^{\gamma u}-1}{e^{\gamma u}} v d \mu \\
\quad=\gamma \int_{\Omega}|\nabla u|^{2} v d x+\int_{\Omega} f v d x \\
\forall v \in H_{0}^{1}(\Omega) \cap L^{\infty}(\Omega) \cap L_{\mu}^{2}(\Omega)
\end{array}\right.
$$

In the case where $\mu$ is a Radon measure, the solution of (0.6) satisfies

$$
\left\{\begin{array}{l}
-\Delta u+\lambda u+\frac{1}{\gamma} \frac{e^{\gamma u}-1}{e^{\gamma u}} \mu=\gamma|\nabla u|^{2}+f \text { in } \mathcal{D}^{\prime}(\Omega) \\
u \in H_{0}^{1}(\Omega) \cap L^{\infty}(\Omega) \cap L_{\mu}^{2}(\Omega)
\end{array}\right.
$$

As in the linear case, there is here a new term $\left(e^{\gamma u}-1\right) \mu /\left(\gamma e^{\gamma u}\right)(\mu$ is the same measure as in the linear case), which depends only on the values of $u$, but it is no more linear. This means that the perturbation of the linear problem $(0.2)$ by a nonlinear term of the form $\gamma\left|\nabla u_{n}\right|^{2}$ changes the structure of the new term in the limit equation.

This result is proved in Section 2 below. The same result is proved in [6] in the case in which the nonlinear perturbation of $(0.2)$ is a general function of the form $H(x, u, \nabla u)$, where $H$ has a (at most) quadratic growth in the gradient variable. In this case the homogenized equation reads as

$$
\left\{\begin{array}{l}
-\Delta u+\lambda u+g(x, u) \mu=f+H(x, u, \nabla u) \text { in } \mathcal{D}^{\prime}(\Omega) \\
u \in H_{0}^{1}(\Omega) \cap L^{\infty}(\Omega) \cap L_{\mu}^{2}(\Omega)
\end{array}\right.
$$

Note that as in $(0.7)$ the nonlinear perturbation $H(x, u, \nabla u)$ remains the same after passing at the limit, but that a new term, $g(x, u) \mu$, appears, which is no more explicit but involves the measure $\mu$ and a new function $g(x, u)$ which results from the interaction of the homogenization and of the nonlinear perturbation. (In this paper, [6], we restrict ourselves for the sake of simplicity to the case where the measure $\mu$ which appears in $(0.3)$ and $(0.4)$ is a Radon measure.) 
In Section 3 below we obtain a corrector resuit, i.e. an approximation (or more exactly a representation) of $\nabla u_{n}$ in the strong topology of $L^{2}(\Omega)^{d}$. Indeed, we establish (see Theorem 3.1) the existence of a sequence of Carathéodory functions $F_{n}: \Omega \times \mathbb{R} \mapsto \mathbb{R}^{d}$ such that

$$
\lim _{n \rightarrow \infty} \int_{\Omega}\left|\nabla u_{n}-\nabla u-F_{n}(x, u)\right|^{2} d x=0 .
$$

For the linear problem (0.2) a similar result is well known to hold (see for example [9], [10], [5]). In this case the functions $F_{n}(x, s)$ are linear in $s$, which is no more the case for problem $(0.1)$.

In Section 4 , we compare the homogenization problem $(0.1)$ (in which the open set $\Omega_{n}$ is varying) with the following homogenization problem (in which the coefficients of the equation are varying)

$$
\left\{\begin{array}{l}
\operatorname{div}\left(A_{n} \nabla u_{n}\right)+\lambda u_{n}=H\left(x, \nabla u_{n}\right) \text { in } \mathcal{D}^{\prime}(\Omega) \\
u_{n} \in H_{0}^{1}(\Omega) \cap L^{\infty}(\Omega),
\end{array}\right.
$$

where $A_{n}=A_{n}(x)$ is a sequence of matrices which H-converges to a matrix $A$ (see [21] for the definition of $H$-convergence) and where $H(x, \xi)$ is a Carathéodory function with a quadratic growth in $\xi$ (for example $\left.H(x, \xi)=f(x)+\gamma|\xi|^{2}, f \in L^{\infty}(\Omega)\right)$.

The homogenization of (0.9) has been studied in [2] (see also [3]). In that case $\left|\nabla u_{n}\right|^{2}$ is equi-integrable and this plays a very important role in the proof. In constrast, the sequence $\left|\nabla u_{n}\right|^{2}$ is not equi-integrable in problem $(0.1)$, which explains why the homogenized problems obtained from $(0.1)$ and $(0.9)$ are very differents.

\section{PRELIMINARIES}

In this Section, we recall some results concerning the homogenization of the linear problem $(0.2)$ in varying domains, which will be used in the next Section to homogenize the quasi-linear problem (0.1). The homogenization of the linear problem $(0.2)$ has been studied by many authors, see for example [9], [11], [10]. The results presented here are mostly due to G. Dal Maso and A. Garroni, [10] (see also [13], [14] and [8]).

Consider a sequence $\Omega_{n}$ of arbitrary open sets which are included in a bounded open set $\Omega$ of $\mathbb{R}^{d}$. In order to define them on the fixed open set $\Omega$, the functions of $H_{0}^{1}\left(\Omega_{n}\right)$ will always be extended by zero in $\Omega \backslash \Omega_{n}$. So, they will be considered as elements of $H_{0}^{1}(\Omega)$. Define the function $w_{n}$ as the solution of the problem

$$
\left\{\begin{array}{l}
-\Delta w_{n}=1 \text { in } \mathcal{D}^{\prime}\left(\Omega_{n}\right) \\
w_{n} \in H_{0}^{1}\left(\Omega_{n}\right)
\end{array}\right.
$$


It is easy to prove that $w_{n}$ is bounded in $H_{0}^{1}(\Omega)$. It can also be proved that

$$
0 \leq w_{n} \leq M \text { a.e. in } \Omega
$$

for some constant $M$. Therefore there exists a subsequence (that for the sake of simplicity, we will still denote by $n$ ) and some $w \in H_{0}^{1}(\Omega)$ such that $w_{n}$ converges weakly in $H_{0}^{1}(\Omega)$ to $w$. Define a distribution $\nu \in H^{-1}(\Omega)$ by

$$
\nu=1+\Delta w .
$$

It can be proved by the maximum principle that the distribution $\nu$ is a nonnegative Radon measure in $\Omega$. Define finally for every Borel set $B \subset \Omega$, the Borel measure $\mu$ by

$$
\mu(B)= \begin{cases}\int_{B} \frac{d \nu}{w} & \text { if } \operatorname{cap}(B \cap\{w=0\})=0 \\ +\infty & \text { if } \operatorname{cap}(B \cap\{w=0\})>0\end{cases}
$$

where $\operatorname{cap}(A)$ denotes the capacity of the set $A$ with respect to $\Omega$, which is defined in the following way: If $A$ is a compact set, the capacity of $A$ is defined by

$$
\operatorname{cap}(A)=\inf \left\{\int_{\Omega}|\nabla \varphi|^{2} d x: \varphi \in \mathcal{D}(\Omega), \varphi \geq \chi_{A}\right\}
$$

If $A$ is an open set, the capacity of $A$ is defined by

$$
\operatorname{cap}(A)=\sup \{\operatorname{cap}(K): K \subset A, K \text { compact }\} .
$$

If $A$ is an arbitrary set, the capacity of $A$ is defined by

$$
\operatorname{cap}(A)=\inf \{\operatorname{cap}(G): A \subset G \subset \Omega, G \text { open }\} .
$$

By definition $\mu$ vanishes on the Borel sets of zero capacity. It is well known (see e.g. [16], [23], [15]) that a function of $H_{0}^{1}(\Omega)$ has a representative which is defined quasi-everywhere (q.e.), i.e. defined except on a set of zero capacity. We will always use this representative for the functions of $H_{0}^{1}(\Omega)$, which are thus defined $\mu$ almost everywhere. It can be shown (see [10]) that the function $w$ and the measure $\mu$ are related by

$$
\left\{\begin{array}{l}
w \in I_{0}^{1}(\Omega) \cap L_{\mu}^{2}(\Omega) \\
\int_{\Omega} \nabla w \nabla v d x+\int_{\Omega \Omega} w v d \mu=\int_{\Omega} v d x \\
\forall v \in H_{0}^{1}(\Omega) \cap L_{\mu}^{2}(\Omega) .
\end{array}\right.
$$


With these definitions, G. Dal Maso and A. Garroni ([10]) have shown the following homogenization result for the linear problem $(0.2)$ :

THEOREM 1.1. - Consider a sequence $f_{n}$ of $H^{-1}(\Omega)$ which converges to some $f$ strongly in $H^{-1}(\Omega)$. Let $u_{n}$ be the solution of the problem

$$
\left\{\begin{array}{l}
-\Delta u_{n}=f_{n} \text { in } \mathcal{D}^{\prime}\left(\Omega_{n}\right) \\
u_{n} \in H_{0}^{1}\left(\Omega_{n}\right)
\end{array}\right.
$$

Then (the whole sequence) $u_{n}$ converges weakly in $H_{0}^{1}(\Omega)$ and strongly in $W^{1, p}(\Omega), 1 \leq p<2$, to the unique solution $u$ of the problem

$$
\left\{\begin{array}{l}
u \in H_{0}^{1}(\Omega) \cap L_{\mu}^{2}(\Omega) \\
\int_{\Omega} \nabla u \nabla v d x+\int_{\Omega} u v d \mu=\langle f, v\rangle \\
\forall v \in H_{0}^{1}(\Omega) \cap L_{\mu}^{2}(\Omega) .
\end{array}\right.
$$

REMARK 1.1. - The problem satisfied by the limit $u$ is similar to the problem satisfied by the function $u_{n}$ but a new term, $\mu u$, appears. This is the "strange term" in the terminology of D. Cioranescu and F. Murat ([9]). However, if, following G. Dal Maso and U. Mosco ([11], [12]), we define for every Borel set $B \subset \Omega$ the measures $\mu_{n}$ by

$$
\mu_{n}(B)= \begin{cases}0 & \text { if } \operatorname{cap}\left(B \cap\left(\Omega \backslash \Omega_{n}\right)\right)=0 \\ +\infty & \text { if } \operatorname{cap}\left(B \cap\left(\Omega \backslash \Omega_{n}\right)\right)>0\end{cases}
$$

problem (1.5) can be written in a form similar to (1.6), i.e.

$$
\left\{\begin{array}{l}
u_{n} \in H_{0}^{1}(\Omega) \cap L_{\mu_{n}}^{2}(\Omega) \\
\int_{\Omega} \nabla u_{n} \nabla v d x+\int_{\Omega} u_{n} v d \mu_{n}=\left\langle f_{n}, v\right\rangle \\
\forall v \in I_{0}^{1}(\Omega) \cap L_{\mu_{n}}^{2}(\Omega) .
\end{array}\right.
$$

Let us conclude this Section by recalling the corrector result for the linear problem (0.2) (or (1.5)). The following theorem has been established in [10] (see also [9], [13], [14], [5] and [8] for related results).

THEOREM 1.2. - Let $u_{n}, u, f_{n}$ and $f$ be as in Theorem 1.1, with $f$ in $L^{\infty}(\Omega)$. Define $R_{n}$ by

$$
\nabla u_{n}= \begin{cases}\nabla u+\frac{u}{w} \nabla\left(w_{n}-w\right)+R_{n} & \text { a.e. on }\{x \in \Omega: w(x)>0\} \\ R_{n} & \text { a.e. on }\{x \in \Omega: w(x)=0\} .\end{cases}
$$

Then the sequence $R_{n}$ converges strongly to zero in $L^{2}(\Omega)^{d}$. 
Remark 1.2. - An important step in the proof of Theorem 1.2, which we will use below, is to note that

$$
|u| \leq\|f\|_{L^{\infty}(\Omega)} w \text { q.e. in } \Omega
$$

a result which is easily proved by the maximum principle, using (1.4) and (1.6). In view of (1.10) it is clear that $\frac{u}{w}$ belongs to $L^{\infty}(\{x \in \Omega: w(x)>$ $0\})$ and thus that $\frac{u}{w} \nabla\left(w_{n}-w\right)$ and $R_{n}$ belong to $L^{2}(\{x \in \Omega: w(x)>0\})$.

From (1.10), we deduce that

$$
\nabla u=0 \text { a.e. in }\{x \in \Omega: w(x)=0\} .
$$

For our purpose, it is better to modify in Theorem 1.2 the sequence $w_{n}$ and to replace it by another sequence $\tilde{w}_{n}$ which is defined by

$$
\tilde{w}_{n}=\min \left\{w_{n}, w\right\}
$$

which has the advantage that

$$
0 \leq \tilde{w}_{n} \leq w \text { q.e. in } \Omega
$$

Using the sequence $\tilde{w}_{n}$, we have

LEMMA 1.1. - Let $u_{n}, u, f_{n}$ and $f$ be as in Theorem 1.2. Then the sequence $\tilde{u}_{n}$ defined by

$$
\tilde{u}_{n}= \begin{cases}u \frac{\tilde{w}_{n}}{w} & \text { q.e. on }\{x \in \Omega: w(x)>0\} \\ 0 & \text { q.e. on }\{x \in \Omega: w(x)=0\}\end{cases}
$$

belongs to $H_{0}^{1}\left(\Omega_{n}\right) \cap L^{\infty}(\Omega)$ and satisfies

(1.15) $\left|\tilde{u}_{n}\right| \leq|u|$ q.e. in $\Omega$

(1.16) $\quad \tilde{u}_{n} u \geq 0$ q.e. in $\Omega$

(1.17) $\nabla \tilde{u}_{n}= \begin{cases}\frac{\tilde{w}_{n}}{w} \nabla u+\frac{u}{w} \nabla \tilde{w}_{n}-\frac{u \tilde{w}_{n}}{w^{2}} \nabla w & \text { a.e. on }\{x \in \Omega: w(x)>0\} \\ 0 & \text { a.e. on }\{x \in \Omega: w(x)=0\} .\end{cases}$

Proof. - From (1.10) and (1.13), it is clear that $\tilde{u}_{n} \in L^{\infty}(\Omega)$ and satisfies (1.15) and (1.16).For $\varepsilon>0$ define $\tilde{u}_{n}^{\varepsilon}$ by

$$
\tilde{u}_{n}^{\varepsilon}=u \frac{\tilde{w}_{n}}{w+\varepsilon} .
$$

Vol. 14, n ${ }^{\circ}$ 5-1997. 
The sequence $\tilde{u}_{n}^{\varepsilon}$ belongs to $H_{0}^{1}\left(\Omega_{n}\right)$ and converges pointwise to $\tilde{u}_{n}$ when $\varepsilon$ tends to zero. Its gradient is

$$
\nabla \tilde{u}_{n}^{\varepsilon}=\frac{\tilde{w}_{n}}{w+\varepsilon} \nabla u+\frac{u}{w+\varepsilon} \nabla \tilde{w}_{n}-\frac{u \tilde{w}_{n}}{(w+\varepsilon)^{2}} \nabla w
$$

By (1.10), (1.13) and the Lebesgue's dominated convergence theorem, $\nabla \tilde{u}_{n}^{\varepsilon}$ converges strongly in $L^{2}(\Omega)^{d}$, when $\varepsilon$ tends to zero, to the expression of $\nabla \tilde{u}_{n}$ given in (1.17). Therefore, we conclude that $\tilde{u}_{n}$ belongs to $H_{0}^{1}\left(\Omega_{n}\right)$ and that its gradient is given by (1.17).

We have now in position to establish the following version of the corrector result (compare with Theorem 1.2).

THEOREM 1.3. - Let $u_{n}, u, f_{n}$ and $f$ be as in Theorem 1.2 and let $\tilde{u}_{n}$ be defined by (1.14). Define $\tilde{r}_{n}$ and $\tilde{R}_{n}$ by

(1.20) $\nabla u_{n}= \begin{cases}\nabla u+\frac{u}{w} \nabla\left(\tilde{w}_{n}-w\right)+\tilde{R}_{n} & \text { a.e. on }\{x \in \Omega: w(x)>0\} \\ \tilde{R}_{n} & \text { a.e. on }\{x \in \Omega: w(x)=0\}\end{cases}$

Then the sequences $\tilde{r}_{n}$ and $\tilde{R}_{n}$ converge strongly to zero in $H_{0}^{1}(\Omega)$ and $L^{2}(\Omega)^{d}$ respectively.

Proof: - Let us first prove that

$$
w_{n}-\tilde{w}_{n} \rightarrow 0 \text { in } H_{0}^{1}(\Omega) .
$$

Since $\tilde{w}_{n}=w_{n}-\left(w_{n}-w\right)^{+}$, it is enough to prove that $\left(w_{n}-w\right)^{+}$ converges to zero in $H_{0}^{1}(\Omega)$. The function $\left(w_{n}-w\right)^{+}$belongs to $H_{0}^{1}\left(\Omega_{n}\right)$ and is thus an admisible test function for (1.1). This yields

$$
\left\{\begin{array}{l}
\int_{\Omega} \nabla w_{n} \nabla\left(w_{n}-w\right)^{+} d x=\int_{\Omega_{n}} \nabla w_{n} \nabla\left(w_{n}-w\right)^{+} d x \\
=\int_{\Omega_{n}}\left(w_{n}-w\right)^{+} d x=\int_{\Omega}\left(w_{n}-w\right)^{+} d x \rightarrow 0
\end{array}\right.
$$

Since $\left(w_{n}-w\right)^{+}$converges weakly to zero in $H_{0}^{1}(\Omega)$ it is also clear that

$$
\int_{\Omega} \nabla w \nabla\left(w_{n}-w\right)^{+} d x \rightarrow 0
$$

Taking the difference of (1.22) and (1.23), we have proved (1.21). 
Let now $u_{n}, u, f_{n}$ and $f$ be as in Theorem 1.2. In view of the definitions (1.9) and (1.20) of $R_{n}$ and $\tilde{R}_{n}$, and of Theorem 1.2, it is enough, in order to prove that $\tilde{R}_{n}$ converges strongly to zero in $L^{2}(\Omega)^{d}$, to show that

$$
\lim _{n \rightarrow \infty} \int_{\{w>0\}} \frac{u^{2}}{w^{2}}\left|\nabla\left(\tilde{w}_{n}-w_{n}\right)\right|^{2} d x=0
$$

which easily follows from (1.10) and (1.21).

Using (1.17) and (1.20), we have

$$
\begin{aligned}
& \nabla\left(u_{n}-\tilde{u}_{n}\right) \\
& = \begin{cases}\nabla u_{n}-\frac{\tilde{w}_{n}}{w} \nabla u-\frac{u}{w} \nabla \tilde{w}_{n}+\frac{u \tilde{w}_{n}}{w^{2}} \nabla w & \text { a.e. in }\{x \in \Omega: w(x)>0\} \\
\nabla u_{n} & \text { a.e. in }\{x \in \Omega: w(x)=0\}\end{cases} \\
& = \begin{cases}\left(1-\frac{\tilde{w}_{n}}{w}\right) \nabla u+\frac{u}{w}\left(\frac{\tilde{w}_{n}}{w}-1\right) \nabla w+\tilde{R}_{n} & \text { a.e. in }\{x \in \Omega: w(x)>0\} \\
\dot{R}_{n} & \text { a.e. in }\{x \in \Omega: w(x)=0\} .\end{cases}
\end{aligned}
$$

By the strong convergence to zero in $L^{2}(\Omega)^{d}$ of $\tilde{R}_{n},(1.10),(1.13)$ and the Lebesgue's dominated convergence theorem, the right-hand side converges strongly in $L^{2}(\Omega)^{d}$ to zero. This proves the strong convergence of $\tilde{r}_{n}$ to zero in $H_{0}^{1}(\Omega)$.

We complete this Section with the following lemma which will be used later.

LEMMA 1.2. - ([8], see also [11], [9], [5]). If $u_{n} \in H_{0}^{1}\left(\Omega_{n}\right)$ converges weakly in $H_{0}^{1}(\Omega)$ to a function $u$, then $u \in L_{\mu}^{2}(\Omega)$.

\section{HOMOGENIZATION OF THE NONLINEAR PROBLEM (0.1)}

In this Section, we use Theorem 1.1 to pass to the limit in $(0.1)$. Assume that $\Omega_{n}$ is such that, for the whole sequence $n$, the solution $w_{n}$ of (1.1) converges weakly in $H_{0}^{1}(\Omega)$ to a function $w$ and define $\mu$ by (1.3).

TheOREM 2.1. - Let $\lambda$, $\gamma$ be real constants with $\lambda>0$. For any $f \in L^{\infty}(\Omega)$, define $u_{n}$ as the unique solution of the problem

$$
\left\{\begin{array}{l}
-\Delta u_{n}+\lambda u_{n}=\gamma\left|\nabla u_{n}\right|^{2}+f \text { in } \mathcal{D}^{\prime}\left(\Omega_{n}\right) \\
u_{n} \in H_{0}^{1}\left(\Omega_{n}\right) \cap L^{\infty}\left(\Omega_{n}\right)
\end{array}\right.
$$


Then, the sequence $u_{n}$ converges weakly in $H_{0}^{1}(\Omega)$, weakly-* in $L^{\infty}(\Omega)$ and strongly in $W^{1, p}(\Omega), 1 \leq p<2$, to the unique solution u of the problem

$$
\left\{\begin{array}{l}
u \in H_{0}^{1}(\Omega) \cap L^{\infty}(\Omega) \cap L_{\mu}^{2}(\Omega) \\
\int_{\Omega} \nabla u \nabla v d x+\lambda \int_{\Omega} u v d x+\frac{1}{\gamma} \int_{\Omega} \frac{e^{\gamma u}-1}{e^{\gamma u}} v d \mu \\
\quad=\gamma \int_{\Omega}|\nabla u|^{2} v d x+\int_{\Omega} f v d x \\
\forall v \in H_{0}^{1}(\Omega) \cap L^{\infty}(\Omega) \cap L_{\mu}^{2}(\Omega) .
\end{array}\right.
$$

Proof. - It has been shown in [4] that (2.1) has a (at least) solution (this solution is proved in [1] to be unique) and that the norm of $u_{n}$ in $H_{0}^{1}\left(\Omega_{n}\right) \cap L^{\infty}\left(\Omega_{n}\right)$ is bounded. Indeed, it follows from an estimate along the lines of the maximum principle (see [4]) that

$$
\left\|u_{n}\right\|_{L^{\infty}\left(\Omega_{n}\right)} \leq \frac{C_{0}}{\lambda}, \text { where } C_{0}=\|f\|_{L^{\infty}(\Omega)}
$$

while the $H_{0}^{1}\left(\Omega_{n}\right)$ estimate is more difficult to state. Actually, in the present case, the change of unknown function (2.4), which will be used below, allows one to retrieve these existence and boundedness results in a simple way. These estimates imply the existence of a subsequence of $n$ (still denoted by $n$ ) such that $u_{n}$ converges weakly in $H_{0}^{1}(\Omega)$ and weakly-* in $L^{\infty}(\Omega)$ to a function $u$, which by Lemma 1.2 belongs to $H_{0}^{1}(\Omega) \cap L_{\mu}^{2}(\Omega) \cap L^{\infty}(\Omega)$. An argument similar to the one used in [1] implies that problem (2.2) has a unique solution. This uniqueness result implies that it is not necessary to extract any subsequence of $n$ whenever $u$ is proved to satisfy (2.2).

Define the function $z_{n}$ by

$$
z_{n}=e^{\gamma u_{n}}-1
$$

Using (2.3), we have the following estimate for $z_{n}$ :

$$
e^{\frac{\gamma c_{0}}{\lambda}} \geq 1+z_{n}=e^{\gamma u_{n}} \geq e^{-\frac{\gamma C_{0}}{\lambda}} \text { a.e. in } \Omega \text {. }
$$

Note that $z_{n} \subset H_{0}^{1}\left(\Omega_{n}\right) \cap L^{\infty}\left(\Omega_{n}\right)$. From

$$
\nabla z_{n}=\gamma e^{\gamma u_{\imath}} \nabla u_{n}
$$

and from (2.4), we deduce that

$$
\nabla u_{n}=\frac{\nabla z_{n}}{\gamma\left(1+z_{n}\right)}
$$


while

$$
u_{n}=\frac{1}{\gamma} \log \left(1+z_{n}\right) .
$$

We therefore deduce from (2.1) that for every $v \in H_{0}^{1}\left(\Omega_{n}\right) \cap L^{\infty}\left(\Omega_{n}\right)$ one has

$$
\begin{aligned}
& { }_{\gamma} \int_{\Omega_{n}} \frac{\nabla z_{n} \nabla v}{\left(1+z_{n}\right)} d x+\frac{\lambda}{\gamma} \int_{\Omega_{n}} \log \left(1+z_{n}\right) v d x \\
& \quad=\frac{1}{\gamma} \int_{\Omega_{n}} \frac{\left|\nabla z_{n}\right|^{2}}{\left(1+z_{n}\right)^{2}} v d x+\int_{\Omega} f v d x .
\end{aligned}
$$

Using $v=\gamma\left(1+z_{n}\right) \varphi$, with $\varphi \in H_{0}^{1}\left(\Omega_{n}\right) \cap L^{\infty}\left(\Omega_{n}\right)$ (this function $v$ belongs to $H_{0}^{1}\left(\Omega_{n}\right) \cap L^{\infty}\left(\Omega_{n}\right)$ ) as test function in (2.7), we obtain

$$
\begin{gathered}
\int_{\Omega_{n}} \nabla z_{n} \nabla \varphi d x=\int_{\Omega_{n}}\left(1+z_{n}\right)\left(\gamma f-\lambda \log \left(1+z_{n}\right)\right) \varphi d x \\
\forall \varphi \in H_{0}^{1}\left(\Omega_{n}\right) \cap L^{\infty}\left(\Omega_{n}\right)
\end{gathered}
$$

Using (2.8) it is now easy to show that $z_{n}$ is bounded in $H_{0}^{1}\left(\Omega_{n}\right)$ (which also follows from $u_{n}$ bounded in $H_{0}^{1}\left(\Omega_{n}\right)$ ). Indeed, taking $\varphi=z_{n}$ in (2.8) gives

$$
\left\{\begin{array}{l}
\int_{\Omega_{n}}\left|\nabla z_{n}\right|^{2} d x+\int_{\Omega_{n}}\left(\lambda \log \left(1+z_{n}\right)-\gamma f\right)\left(1+z_{n}\right)^{2} d x \\
=\lambda \int_{\Omega_{n}}\left(1+z_{n}\right) \log \left(1+z_{n}\right) d x-\gamma \int_{\Omega_{n}} f\left(1+z_{n}\right) d x
\end{array}\right.
$$

which, together with (2.5) implies that $\left\|z_{n}\right\|_{H_{0}^{1}(\Omega)}=\left\|z_{n}\right\|_{H_{0}^{1}\left(\Omega_{n}\right)}$ is bounded.

Rellich-Kondrachov's theorem, (2.5), and Lebesgue's dominated convergence theorem imply that the sequence $\left(1+z_{n}\right)\left(\gamma f-\lambda \log \left(1+z_{n}\right)\right)$ converges strongly in $L^{2}(\Omega)$ to the function $(1+z)(\gamma f-\lambda \log (1+z))$, where $z=e^{\gamma u}-1$ (see (2.4)). Therefore Theorem 1.1 applied to (2.8) implies that the function $z$ is solution of

$$
\left\{\begin{array}{l}
z \in I I_{0}^{1}(\Omega) \cap L^{\infty}(\Omega) \cap L_{\mu}^{2}(\Omega) \\
\int_{\Omega \Omega} \nabla z \nabla v d x+\int_{\Omega} z v d \mu=\int_{\Omega}(1+z)(\gamma f-\lambda \log (1+z)) v \\
\forall v \in H_{0}^{1}(\Omega) \cap L_{\mu}^{2}(\Omega) .
\end{array}\right.
$$

Using $v=\frac{\varphi}{\gamma(1+z)}$ with $\varphi \in H_{0}^{1}(\Omega) \cap L^{\infty}(\Omega) \cap L_{\mu}^{2}(\Omega)$ as test function in (2.9) implies that $u$ satisfies (2.2), which finishes the proof.

Vol. 14, $n^{\circ}$ 5-1997. 
Remark 2.1. - If $\gamma=0$, Theorem 1.1 applied to Problem (2.1) implies that $u$ satisfies

$$
\left\{\begin{array}{l}
u \in H_{0}^{1}(\Omega) \cap L_{\mu}^{2}(\Omega) \\
\int_{\Omega} \nabla u \nabla v d x+\lambda \int_{\Omega} u v d x+\int_{\Omega} u v d \mu=\int_{\Omega} f v d x \\
\forall v \in H_{0}^{1}(\Omega) \cap L_{\mu}^{2}(\Omega)
\end{array}\right.
$$

In fact

$$
\lim _{\gamma \rightarrow 0} \frac{1}{\gamma} \frac{e^{\gamma u}-1}{e^{\gamma u}}=u
$$

and Theorem 2.1 is thus consistent with Theorem 1.1.

\section{CORRECTOR}

The aim of this Section is to prove the following corrector result.

THEOREM 3.1. - In the framework of Theorem 2.1, let $u_{n}, u, w_{n}, \tilde{w}_{n}$ and $w$ be respectively defined by (2.1), (2.2), (1.1), (1.12) and (1.4). Define $R_{n}$ and $r_{n}$ by

$$
\begin{aligned}
& \text { (3.1) } u_{n}= \begin{cases}\frac{1}{\gamma} \log \left(1+\frac{\check{u}_{n}}{w}\left(e^{\gamma u}-1\right)\right)+r_{n} & \text { q.e. on }\{x \in \Omega: w(x)>0\} \\
r_{n} & \text { q.e. on }\{x \in \Omega: w(x)=0\}\end{cases} \\
& \text { (3.2) } \nabla u_{n}= \begin{cases}\nabla u+\frac{1}{\gamma} \frac{\sigma^{\gamma u}-1}{w+\tilde{w}_{n}\left(\gamma^{\gamma u}-1\right)} \nabla\left(\tilde{u}_{n}-w\right)+R_{n} & \text { a.e. on }\{x \in \Omega: w(x)>0\} \\
R_{n} & \text { a.e. on }\{x \in \Omega: w(x)=0\} .\end{cases}
\end{aligned}
$$

Then the sequences $r_{n}$ and $R_{n}$ converge strongly to zero in $H_{0}^{1}(\Omega)$ and $L^{2}(\Omega)^{d}$ respectively.

REMARK 3.1. - From (1.13) and (2.5), we deduce that

$$
1+\frac{\tilde{w}_{n}}{w} z=1+\frac{\tilde{w}_{n}}{w}\left(e^{\gamma u}-1\right) \geq 1+\frac{\tilde{w}_{n}}{w}\left(e^{-\frac{\gamma C_{0}}{\lambda}}-1\right) \geq e^{-\frac{\gamma C_{0}}{\lambda}} \text { q.e. in } \Omega \text {. }
$$

Therefore, the logarithm in (3.1) and the denominator in (3.2) are well defined. Here the use of $\tilde{w}_{n}$ in place of $w_{n}$ proves to be useful.

Proof of Theorem 3.1. - To simplify the notation, let us denote by $O_{n}$ any sequence of functions of $L^{2}(\Omega)^{d}$ which can change from a line to another, such that $O_{n}$ converges strongly to zero in $L^{2}(\Omega)^{d}$. We want to prove in particulat that $R_{n}$ defined by (3.2) is such an $O_{n}$. 
First step. - Since $z_{n}$ satisfies (2.8), and since $\left(1+z_{n}\right)\left(\gamma f-\lambda \log \left(1+z_{n}\right)\right)$ is bounded in $L^{\infty}(\Omega)$, Theorem 1.3 implies that defining $\tilde{r}_{n}$ and $\tilde{R}_{n}$ by

$$
\begin{aligned}
& \text { (3.4) } z_{n}= \begin{cases}z \frac{\tilde{w}_{n}}{w}+\tilde{r}_{n} & \text { q.e. on }\{x \in \Omega: w(x)>0\} \\
\tilde{r}_{n} & \text { q.e. on }\{x \in \Omega: w(x)=0\}\end{cases} \\
& (3.5) \nabla z_{n}= \begin{cases}\nabla z+\frac{z}{w} \nabla\left(\tilde{w}_{n}-w\right)+\tilde{R}_{n} & \text { a.e. on }\{x \in \Omega: w(x)>0\} \\
\tilde{R}_{n} & \text { a.e. on }\{x \in \Omega: w(x)=0\},\end{cases}
\end{aligned}
$$

the sequences $\tilde{r}_{n}$ and $\tilde{R}_{n}$ converge strongly to zero in $H_{0}^{1}(\Omega)$ and $L^{2}(\Omega)^{d}$ respectively. By $(1.10)$, we also know that there exists a constant $C>0$ such that

$$
|z| \leq C w \text { q.e. in } \Omega
$$

and by Lemma 1.1 , that the function $\tilde{z}_{n}$ defined by

$$
\tilde{z}_{n}= \begin{cases}z \frac{\tilde{w}_{n}}{w} & \text { q.e. on }\{x \in \Omega: w(x)>0\} \\ 0 & \text { q.e. on }\{x \in \Omega: w(x)=0\}\end{cases}
$$

belongs to $H_{0}^{1}(\Omega) \cap L^{\infty}(\Omega)$.

Using (2.6), and taking into account (2.5), we deduce from (3.5) that on $\{x \in \Omega: w(x)>0\}$

$$
\left\{\begin{aligned}
\nabla u_{n} & =\frac{1}{\gamma} \frac{\nabla z_{n}}{1+z_{n}}=\frac{1}{\gamma} \frac{1}{1+z_{n}}\left(\nabla z+\frac{z}{w} \nabla\left(\tilde{w}_{n}-w\right)+\tilde{R}_{n}\right) \\
& =\frac{1}{\gamma} \frac{\nabla z}{1+z}+\frac{1}{\gamma} \frac{1}{1+z_{n}} \frac{z}{w} \nabla\left(\tilde{w}_{n}-w\right)+O_{n} \\
& =\nabla u+\frac{1}{\gamma} \frac{1}{1+z \frac{\tilde{w}_{n}}{w}+\tilde{r}_{n}} \frac{z}{w} \nabla\left(\tilde{w}_{n}-w\right)+O_{n}
\end{aligned}\right.
$$

while on $\{x \in \Omega: w(x)=0\}$

$$
\nabla u_{n}=\frac{1}{\gamma} \frac{\nabla z_{n}}{1+z_{n}}=\frac{1}{\gamma} \frac{\tilde{R}_{n}}{1+z_{n}}=O_{n} .
$$

Second step. - We will now improve (3.7) by removing $\tilde{r}_{n}$. Indeed, in order to prove that $R_{n}$ defined by (3.2) converges strongly to zero in $L^{2}(\Omega)^{d}$, it is enough to show that

$$
\lim _{n \rightarrow \infty} \int_{\{w>0\}}\left|\left(\frac{1}{1+z \frac{\tilde{w}_{n}}{w}+\tilde{r}_{n}}-\frac{1}{1+z \frac{\tilde{w}_{n}}{w}}\right) \frac{z}{w} \nabla\left(\tilde{w}_{n}-w\right)\right|^{2} d x=0 .
$$


By (3.6), (2.5) and (3.3) we have on $\{x \in \Omega: w(x)>0\}$

$$
\frac{|z|}{w} \leq C, \quad 1+z \frac{\tilde{w}_{n}}{w}+\tilde{r}_{n}=1+z_{n} \geq e^{\frac{-\gamma C_{0}}{\lambda}}, 1+z \frac{\tilde{w}_{n}}{w} \geq e^{\frac{-\gamma C_{0}}{\lambda}} .
$$

Thus

$$
\begin{aligned}
& \limsup _{n \rightarrow \infty} \int_{\{w>0\}}\left|\left(\frac{1}{1+z \frac{\tilde{w}_{n}}{w}+\tilde{r}_{n}}-\frac{1}{1+z \frac{\tilde{w}_{n}}{w}}\right) \frac{z}{w} \nabla\left(\tilde{w}_{n}-w\right)\right|^{2} d x \\
& \quad<\limsup _{n \rightarrow \infty} C^{2} e^{\frac{4 \gamma C_{0}}{\lambda}} \int_{\Omega} \tilde{r}_{n}^{2}\left|\nabla\left(\tilde{w}_{n}-w\right)\right|^{2} d x \\
& \quad=\limsup _{n \rightarrow \infty} C^{2} e^{\frac{4 \gamma c_{0}}{\lambda}} \int_{\Omega} \tilde{r}_{n}^{2}\left|\nabla \tilde{w}_{n}\right|^{2} d x,
\end{aligned}
$$

where in the last equality, we use the fact that the sequence $\tilde{r}_{n}$ is bounded in $L^{\infty}(\Omega)$. It is then enough to show that

$$
\lim _{n \rightarrow \infty} \int_{\Omega} \tilde{r}_{n}^{2}\left|\nabla \tilde{w}_{n}\right|^{2} d x=0 .
$$

Third step. - Proof of (3.8).

Since $\tilde{w}_{n}$ and $\tilde{r}_{n}$ belong to $H_{0}^{1}\left(\Omega_{n}\right) \cap L^{\infty}\left(\Omega_{n}\right)$, the function $\tilde{r}_{n}^{2} \tilde{w}_{n}$ belongs to $H_{0}^{1}\left(\Omega_{n}\right)$. We can therefore use it as test function in (1.1) or more exactly in

$$
-\Delta \tilde{w}_{n}=1-\Delta\left(\tilde{w}_{n}-w_{n}\right) \text { in } \mathcal{D}^{\prime}\left(\Omega_{n}\right)
$$

We obtain

$$
\begin{aligned}
& \int_{\Omega_{n}}\left|\nabla \tilde{w}_{n}\right|^{2} \tilde{r}_{n}^{2} d x+2 \int_{\Omega_{n}} \tilde{r}_{n} \tilde{w}_{n} \nabla \tilde{w}_{n} \nabla \tilde{r}_{n} d x \\
& =\int_{\Omega_{n}} \tilde{r}_{n}^{2} \tilde{m}_{n} d x+\int_{\Omega_{n}} \nabla\left(\tilde{w}_{n}-w_{n}\right) \nabla\left(\tilde{r}_{n}^{2} \tilde{\mu}_{n}\right) d x .
\end{aligned}
$$

Using Rellich-Kondrachov's theorem, the fact that $\tilde{w}_{n}$ and $\tilde{r}_{n}$ are bounded in $L^{\infty}(\Omega)$ and that $\tilde{r}_{n}$ and $\tilde{w}_{n}-w_{n}$ converge strongly to zero in $H_{0}^{1}(\Omega)$ (see (1.21)), we easily deduce that the second, third and fourth terms tend to zero. This gives (3.8). We have thus proved that $R_{n}$ defined by (3.2) converges to zero strongly in $L^{2}(\Omega)^{d}$.

Fourth step. - Let us now prove that the sequence $r_{n}$ defined by (3.1) converges strongly to zero in $H_{0}^{1}(\Omega)$. 
By (3.3) we have $1+\frac{\tilde{w}_{n}}{w} z \geq e^{\frac{-\gamma C_{0}}{\lambda}}$ quasi everywhere on $\{x \in \Omega$ : $w(x)>0\}$, thus $\log \left(1+\frac{\tilde{w}_{n}}{w} z\right)$ is well defined. By Lemma 1.1 the sequence $v_{n}$ defined by

$$
v_{n}= \begin{cases}\frac{1}{\gamma} \log \left(1+\frac{\tilde{w}_{n}}{w} z\right) & \text { q.c. on }\{x \in \Omega: w(x)>0\} \\ 0 & \text { q.e. on }\{x \in \Omega: w(x)=0\}\end{cases}
$$

belongs to $H_{0}^{1}(\Omega) \cap L^{\infty}(\Omega)$ and its gradient is given by

$$
\nabla v_{n}= \begin{cases}\frac{1}{\gamma} \frac{1}{1+\frac{\tilde{w}_{n}}{w} z}\left(\frac{\tilde{w}_{n}}{w} \nabla z+\frac{\Sigma}{w} \nabla \tilde{w}_{n}-\frac{\tilde{u}_{n} z}{w^{2}} \nabla w\right) & \text { a.e. on }\{x \in \Omega: w(x)>0\} \\ 0 & \text { a.e. on }\{x \in \Omega: w(x)=0\} .\end{cases}
$$

From $r_{n}=u_{n}-v_{n}$ it follows that $r_{n} \in H_{0}^{1}(\Omega) \cap L^{\infty}(\Omega)$. As $\nabla r_{n}=\nabla u_{n}=R_{n}$ almost everywhere in $\{x \in \Omega: w(x)=0\}$, we deduce that $\nabla r_{n}$ converges strongly to zero in $L^{2}(\{x \in \Omega: w(x)=0\})^{d}$.

On the other hand, on $\{x \in \Omega: w(x)>0\}$, we use $r_{n}=u_{n}-v_{n}$, (3.10), (3.2), then (1.21), (3.3) and (3.6) and finally (1.13); we obtain

$$
\begin{aligned}
\nabla r_{n} & -\nabla u_{n}-\frac{1}{\gamma} \frac{1}{1+\frac{\tilde{w}_{n}}{w} z}\left(\frac{\tilde{w}_{n}}{w} \nabla z+\frac{z}{w} \nabla \tilde{w}_{n}-\frac{\tilde{w}_{n} z}{w^{2}} \nabla w\right) \\
& =\nabla u-\frac{1}{\gamma} \frac{1}{1+\frac{\tilde{w}_{n}}{w} z} \frac{\tilde{w}_{n}}{w} \nabla z+\frac{1}{\gamma} \frac{1}{1+\frac{\tilde{w}_{n}}{w} z} \frac{z}{w}\left(\frac{\tilde{w}_{n}}{w}-1\right) \nabla w+O_{n} \\
& =\nabla u-\frac{1}{\gamma} \frac{1}{1+z} \nabla z+O_{n}=O_{n}
\end{aligned}
$$

which completes the proof of Theorem 3.1.

\section{COMPARISON WITH THE ANALOGOUS PROBLEM WITH OSCILLATING COEFFICIENTS}

In this Section, we compare the results obtained in the above Sections 2 and 3 with the results obtained by A. Bensoussan, L. Boccardo and F. Murat [2] for the analogous problem with varying coefficients (see also [3]).

Consider a bounded open set $\Omega \subset \mathbb{R}^{d}$ and a Carathéodory function $H: \Omega \times \mathbb{R}^{d} \mapsto \mathbb{R}$ such that for almost every $x \in \Omega$ we have

$$
|H(x, \xi)| \leq C\left(1+|\xi|^{2}\right), \forall \xi \in \mathbb{R}^{d}
$$

where $C$ is a positive constant; the model example is the case $H(x, \xi)=$ $C_{0}+\gamma|\xi|^{2}$. 
We consider the problem

$$
\left\{\begin{array}{l}
-\operatorname{div}\left(A_{n} \nabla u_{n}\right)+\lambda u_{n}=H\left(x, \nabla u_{n}\right) \text { in } \mathcal{D}^{\prime}(\Omega) \\
u_{n} \in H_{0}^{1}(\Omega) \cap L^{\infty}(\Omega) .
\end{array}\right.
$$

where $\lambda>0$ and $A_{n}=A_{n}(x)$ is a sequence of matrices which satisfy $A_{n} \geq \alpha I,\left(A_{n}\right)^{-1} \geq \beta I,(\alpha, \beta>0)$, and which $\mathrm{H}$-converges to a matrix $A$ (see [21] for the definition of $H$-convergence). In constrast with problem (2.1), here the domain is fixed and it is the operator $-\operatorname{div}\left(A_{n} \nabla\right)$ which varies. It has been proved in [4] that there exists a solution of (4.1) which is bounded in $H_{0}^{1}(\Omega) \cap L^{\infty}(\Omega)$ independently of $n$. This solution is moreover unique, (see [1]). Therefore, we can suppose (extracting a subsequence if necessary) that the sequence $u_{n}$ converges weakly to a function $u$ in $H_{0}^{1}(\Omega)$ and weakly-* in $L^{\infty}(\Omega)$.

Following [2] define now $\bar{u}_{n}$ as the solution of the problem

$$
\left\{\begin{array}{l}
-\operatorname{div}\left(A_{n} \nabla \bar{u}_{n}\right)=-\operatorname{div}(A \nabla u) \text { in } \mathcal{D}^{\prime}(\Omega) \\
\bar{u}_{n} \in H_{0}^{1}(\Omega) .
\end{array}\right.
$$

It has been proved in [2] that

$$
u_{n}-\bar{u}_{n} \rightarrow 0 \text { in } H_{0}^{1}(\Omega) \text { strongly. }
$$

This means that the corrector for the linear problem (4.2) is still a corrector for the nonlinear problem (4.1). The proof of (4.3) is based on the fact that $\left|\nabla \bar{u}_{n}\right|^{2}$ is equi-integrable because of Meyers' regularity theorem (see [20] or the appendix of [22]). This implies that $\left|\nabla u_{n}\right|^{2}$ is also equi-integrable. As a result of this, the limit problem of (4.1) reads as

$$
\left\{\begin{array}{l}
-\operatorname{div}(A \nabla u)+\lambda u=\hat{H}(x, \nabla u) \quad \text { in } \mathcal{D}^{\prime}(\Omega), \\
u \in H_{0}^{1}(\Omega) \cap L^{\infty}(\Omega) .
\end{array}\right.
$$

Note that in (4.4) the limit operator - div $(A \nabla)$ is the same as in the linear case, but that the perturbation $\hat{H}(x, \nabla u)$ is no more $H(x, \nabla u)$ in general.

In the case of varying open sets $\Omega_{n}$ that we considered in Sections 2 and 3, the result is different: the nonlinear perturbation $H(x, \nabla u)$ (which was there $\gamma|\nabla u|^{2}$ ) remains the same, but the limit operator $-\Delta u+\left(e^{\gamma u}-1\right) \mu /\left(\gamma e^{\gamma u}\right)$ is no more the operator which appears in the linear case $(\gamma=0)$ where it is $-\Delta u+\mu u$. This is due to the fact that in the nonlinear case $(\gamma \neq 0)$ the corrector result (3.2) really differs from the corrector result (1.9) or (1.20) of the linear case. It should also be emphasized that a careful study of the corrector result (3.2) shows that $\left|\nabla u_{n}\right|^{2}$ is not equi-integrable in general. This is due to the fact that $\left|\nabla w_{n}\right|^{2}$ (and thus $\left|\nabla u_{n}\right|^{2}$ ) is not equi-integrable in general, as it can be proved by considering special examples (see [9]). 


\section{ACKNOWLEDGEMENTS}

I thank François Murat for interesting discussions concerning this paper.

This work has been partially supported by the Project EURHomogenization, Contrat SC1-CT91-0732 of the Program SCIENCE of the Commission of the European Communities and by the Project PB92-0696 of the DGICYT of Spain.

\section{REFERENCES}

[1] G. Barles and F. Murat, Uniqueness and the maximum principle for quasilinear elliptic equations with quadratic growth conditions. Arch. Rat. Mech. Anal., Vol. 133, 1, 1995 , pp. 77-101.

[2] A. Bensoussan, L. Boccardo and F. Murat, $H$-Convergence for quasilinear elliptic equations with quadratic growth, Appl. Math. Optim., Vol. 26, 3, 1992, pp. 253-272.

[3] A. Bensoussan, L. Boccardo, A. Dall aglio and F. Murat, $H$-convergence for quasilinear elliptic equations under natural hypotheses on the correctors. In Composite Media and Homogenization Theory II (Proceedings, Trieste, September 1993), ed. by G. DAL Maso, G. F. Dell'Antonio, 1995, World Scientific, Singapore, pp. 93-112.

[4] L. Boccardo, F. Murat and J.-P. PuEl, Existence de solutions faibles pour des equations elliptiques quasi-linéaires à croissance quadratique. In Nonlinear Partial Differential Equations and their Applications, Collège de France Seminar, Vol. IV, ed. by H. Brézis, J.-L. Lions. Research Notes in Math., Vol. 84, 1983, Pitman, London, pp. 19-73.

[5] J. CaSAdo-Díaz, Sobre la homogeneización de problemas no coercivos y problemas en dominios con agujeros. Ph. D. Thesis, University of Seville, 1993.

[6] J. CASADO-DIAZ, Homogenization of general quasi-linear Dirichlet problems with quadratic growth in perforated domains. To appear in J. Math. Pur. App.

[7] J. CASADO-DíaZ, Homogenization of Dirichlet problems for monotone operators in varying domains. To appear in P. Roy. Soc. Edimburgh.

[8] J. Casado-Díaz and A. GarRoni, Asymptotic behaviour of Dirichlet solutions of nonlinear elliptic systems in varying domains. To appear.

[9] D. Cioranescu and F. Murat, Un terme étrange venu d'ailleurs. In Nonlinear Partial Differential Equations and their Applications, Collège de France Seminar, Vol. II and III, ed. by H. BrÉzIS, J.-L. Lions. Research Notes in Math. Vol. 60 and 70, 1982, Pitman, London, pp. 98-138 and pp. 154-178.

[10] G. Dal Maso and A. Garroni, New results on the asymptotic behaviour of Dirichlet problems in perforated domains, Math. Mod. Meth. Appl. Sci., Vol. 3, 1994, pp. 373-407.

[11] G. Dal Maso and U. Mosco, Wiener criterion and $\Gamma$-convergence. Appl. Math. Optim. Vol. 15 1987, pp. 15-63.

[12] G. Dal MAso and U. Mosco, Wiener-criteria and energy decay for relaxed Dirichlet problems. Arch. Rat. Mech. Anal., Vol. 95, 4, 1986, pp. 345-387.

[13] G. Dal Maso and F. Murat, Dirichlet problems in perforated domains for homogeneous monotone operators on $H_{0}^{1}$. In Calculus of Variations, Homogenization and Continuum Mechanics (Proceedings, Cirm-Lumigny, Marseille, June 2I-25, 1993), ed. by G. Bouchitté, G. Buttazzo, P. Suquet. Series on Advances in Mathematics for Applied Sciences 18, World Scientific, Singapore, 1994, pp. 177-202.

[14] G. Dal Maso and F. Murat, Asymptotic behaviour and correctors for Dirichlet problems in perforated domains with homogeneous monotone operators. To appear.

[15] L. C. Evans and R. F. Gariepy, Measure theory and fine properties of functions. Studies in Advanced Mathematics., CRC Press, Boca Raton, 1992.

Vol. $14, n^{\circ} 5-1997$. 
[16] H. FEDERER and W. ZIEMER, The Lebesgue set of a function whose distribution derivatives are p-th power summable. Indiana U. Math. J., Vol. 22, 1972, 139-158.

[17] S. Finzi Vita, F. Murat and N. A. Tchou, Quasilinear relaxed Dirichlet problems. Siam J. Math. Anal., Vol. 27, 4, 1996, pp. 977-996.

[18] S. FinzI VITA and N. A. Tchou, Corrector results for relaxed Dirichlet problems. Asymptotic Analysis, Vol. 5, 1992, pp. 269-281.

[19] H. Kacimi and F. Murat, Estimation de l'erreur dans des problèmes de Dirichlet où apparaît un terme étrange. In Partial Differential Equations and the Calculus of Variations, Vol. II. Essays in honor of E. De Giorgi, ed. by F. Colombini, L. Modica, A. Marino, S. Spagnolo. Progress in Nonlinear Differential Equations and their Applications 2 , Birkhäuser, Boston, 1989, pp. 661-696.

[20] N. G. Meycrs, An $L^{p}$ estimate for the gradient of solutions of second order elliptic divergence equations. Ann. Sc. Norm. Sup. Pisa C. Sci., Vol. 3, 17, 1963, pp. 189-206.

[21] F. MuRat, $H$-convergence. Séminaire d'Analyse Fonctionnelle et Numérique de l'Université d'Alger 1977/1978, mimeographed notes. Fnglish translation: F. Murat, L. TARTAR, H-convergence. In Mathematical Modelling of Composites Materials, ed. by R. V. KoHN. Progress in Nonlinear Differential Equations and their Applications, Birkhäuser, Boston. To appear.

[22] F. MuRat, Homogenization of renormalized solutions of elliptic equations. Ann. Inst. H Poincaré, Anal. non linéaire, Vol. 8, 1991, pp. 309-332.

[23] W. P. ZiEMER, Weakly differentiable functions. Springer-Verlag, New York, 1989.

(Manuscript received October 27, 1995; revised March 28, 1996.) 